\title{
Conformal Vector Fields on Doubly Warped Product Manifolds and Applications
}

\author{
H. K. El-Sayied, ${ }^{1}$ Sameh Shenawy, ${ }^{2}$ and Noha Syied ${ }^{2}$ \\ ${ }^{1}$ Mathematics Department, Faculty of Science, Tanta University, Tanta 31527, Egypt \\ ${ }^{2}$ Modern Academy for Engineering and Technology, Maadi 11585, Egypt \\ Correspondence should be addressed to Noha Syied; drnsyied@mail.com
}

Received 3 July 2016; Revised 29 August 2016; Accepted 19 September 2016

Academic Editor: Andrei Moroianu

Copyright (c) 2016 H. K. El-Sayied et al. This is an open access article distributed under the Creative Commons Attribution License, which permits unrestricted use, distribution, and reproduction in any medium, provided the original work is properly cited.

\begin{abstract}
This article aimed to study and explore conformal vector fields on doubly warped product manifolds as well as on doubly warped spacetime. Then we derive sufficient conditions for matter and Ricci collineations on doubly warped product manifolds. A special attention is paid to concurrent vector fields. Finally, Ricci solitons on doubly warped product spacetime admitting conformal vector fields are considered.
\end{abstract}

\section{An Introduction}

Bishop and O’Neill introduced Riemannian warped products to construct manifolds with negative sectional curvature [1]. Since then warped product structures have been widely studied. Doubly warped products are generalizations of singly warped products. Beem, Ehrilish, and Powell noticed that there are many exact solutions to Einstein's field equation in the form of warped product manifolds. Since then singly and doubly warped product manifolds have became more indispensable to physicians and mathematicians than ever. In [2], Beem and Powell studied Lorentzian doubly warped product manifolds. Allison studied causal properties, pseudocovexity, and hyperbolicity of doubly warped product manifolds $[3,4]$. Gebarowski considered doubly warped products with harmonic Weyl conformal curvature tensor in [5] and conformally flat and conformally recurrent doubly warped product manifolds in $[6,7]$. Ünal studied geodesic completeness of Riemannian and Lorentzian doubly warped products [8]. He also studied hyperbolicity of generalized Robertson-Walker spacetime with doubly warped product fibre. In this paper, Ünal finally considered some results about conformal vector fields of doubly warped products. Doubly warped product submanifolds have also been studied by many authors in various settings such as Faghfouri and
Majidi in [9], Olteanu in [10, 11], Perktas and Kilic in [12], and many others. Doubly warped spacetime is good example of Lorentzian doubly warped product manifolds. This spacetime is of interest since it produces many exact solutions to Einstein's field equations.

In physics, symmetry assumptions are used to understand the relation between geometry and matter of spacetime given by Einstein's field equation. For example, the metric tensor of (pseudo-)Riemannian manifold does not change under the flow of a Killing vector field; that is, the flow of a Killing vector field generates spacetime symmetry. The number of independent Killing vector fields measures the degree of symmetry of a (pseudo-)Riemannian manifold. Conformal vector fields have also a well-known geometrical and physical interpretations and have been studied on (pseudo-)Riemannian manifolds for a long time. The existence of a conformal vector field on spacetime is especially useful to study its geometry. The flow of a conformal vector consists of conformal transformations of the Riemannian manifold. Thus, the problems of existence and characterization of different types of conformal vector fields in different spaces are important and are widely discussed by both mathematicians and physicists (e.g., see [13-18] and further references contained therein).

The aim of the present paper is to study and explore conformal vector fields on doubly warped product manifolds 
as well as doubly warped spacetime. We derive many characterizations of conformal vector fields on doubly warped product manifolds and doubly warped spacetime. Then, we study matter and Ricci collineation on doubly warped manifolds. One may notice that after Pereleman used Ricci soliton to solve the Poincaré conjecture posed in 1904, a growing body of research has continued to study Ricci soliton. Accordingly, we study Ricci solitons on doubly warped product spacetime admitting many types of conformal vector fields. We get some partial answers of the following questions: What do doubly warped Ricci soliton factors inherit? And what are the conditions under which doubly warped spacetime is a doubly warped Ricci soliton?

This article is organized as follows. Section 2 represents some connection and curvature related formulas on doubly warped product manifolds. In Section 3, we study conformal vector fields on doubly warped product manifolds. Then we study conformal and concurrent vector fields on doubly warped spacetime in two subsections. Finally, Section 4 comprises a study of Ricci soliton on doubly warped spacetime admitting these types of vector fields. Almost all considerations and statements in this work are local.

\section{Preliminaries}

This section represents connection and curvature related formulas on doubly warped product manifolds as a generalization of similar results on singly warped products $[1,19]$. Also, we will provide basic definitions and properties of conformal vector fields.

Let $\left(M_{i}, g_{i}, D_{i}\right)$ be two (pseudo-)Riemannian manifolds with metrics $g_{i}$ and Levi-Civita connections $D_{i}$ and let $f_{i}$ : $M_{i} \rightarrow(0, \infty)$ be a positive function, where $i=1,2$. Also, suppose that $\pi_{i}: M_{1} \times M_{2} \rightarrow M_{i}$ is the natural projection map of the Cartesian product $M_{1} \times M_{2}$ onto $M_{i}$, where $i=1,2$. The (pseudo-)Riemannian manifolds doubly warped product manifold $M={ }_{f_{2}} M_{1} \times{ }_{f_{1}} M_{2}$ is the product manifold $M=M_{1} \times M_{2}$ furnished with the metric tensor

$$
g=\left(f_{2} \circ \pi_{2}\right)^{2} \pi_{1}^{*}\left(g_{1}\right) \oplus\left(f_{1} \circ \pi_{1}\right)^{2} \pi_{2}^{*}\left(g_{2}\right),
$$

where * denotes the pull-back operator on tensors. The functions $f_{i}, i=1,2$, are called the warping functions of the warped product manifold $M$. In particular, if, for example, $f_{2}=1$, then $M=M_{1} \times_{f_{1}} M_{2}$ is called a (singly) warped product manifold. A singly warped product manifold $M_{1} \times{ }_{f_{1}} M_{2}$ is said to be trivial if the warping function $f_{1}$ is also constant $[6,8,9,12,20,21]$. It is clear that the submanifolds $M_{1} \times\{q\}$ and $\{p\} \times M_{2}$ are homothetic to $M_{1}$ and $M_{2}$, respectively, for each $p \in M_{1}$ and $q \in M_{2}$. We shall refer to these factor submanifolds as $M_{1}$ and $M_{2}$. The lift $\bar{X}_{(p, q)}$ of a tangent vector $X_{p} \in T_{p} M_{1}, q \in M_{2}$, is the unique vector in $T_{(p, q)} M$ such that

$$
\begin{aligned}
& \pi_{1}^{*}\left(\bar{X}_{(p, q)}\right)=X_{p}, \\
& \pi_{2}^{*}\left(\bar{X}_{(p, q)}\right)=0 .
\end{aligned}
$$

Similarly, if $X_{i} \in \mathfrak{X}\left(M_{i}\right)$, then the lift of $X_{i}$ to $\mathfrak{X}\left(M_{1} \times M_{2}\right)$ is the unique vector field in $\mathfrak{X}\left(M_{1} \times M_{2}\right)$, that is, $\pi_{i}$ related to $X_{i}$ and $\pi_{j}$ related to zero vector field in $\mathfrak{X}\left(M_{j}\right), i \neq j$; that is, a vector field $X_{i}$ on $M_{i}$ is identified with the horizontal or the vertical vector field on $M_{1} \times M_{2}$, that is, $\pi_{i}$ related to $X_{i}$. Throughout this article we use the same notation for a vector field and for its lift to the product manifold. A function $\omega_{i}$ on $M_{i}$ will be identified with $\omega_{i} \circ \pi_{i}$. Thus, we have two different meanings for the gradient of $\omega_{i}$, namely, grad $\left(\omega_{i} \circ\right.$ $\left.\pi_{i}\right) \in \mathfrak{X}\left(M_{1} \times M_{2}\right)$ and the lift of the gradient $\nabla^{i} \omega_{i}$ of $\omega_{i}$ to $\mathfrak{X}\left(M_{1} \times M_{2}\right)$. In fact, we have

$$
\begin{aligned}
g\left(X_{i}, \operatorname{grad}\left(\omega_{i} \circ \pi_{i}\right)\right) & =X_{i}\left(\omega_{i} \circ \pi_{i}\right)=X_{i}\left(\omega_{i}\right) \circ \pi_{i} \\
& =\frac{1}{f_{j}^{2}} g\left(X_{i}, \nabla^{i} \omega_{i}\right) .
\end{aligned}
$$

Therefore, grad $\left(\omega_{i} \circ \pi_{i}\right)=\left(1 / f_{j}^{2}\right) \nabla^{i} \omega_{i}$ (note that we use the same notation for the vector field $\nabla^{i} \omega_{i}$ and for its lift to $\left.\mathfrak{X}\left(M_{1} \times M_{2}\right)\right)$.

Let $(M, g, D)$ be a pseudo-Riemannian doubly warped product manifold of $\left(M_{i}, g_{i}, D_{i}\right), i=1,2$, with dimensions $n_{i}$, where $n=n_{1}+n_{2} . R, R^{i}$ and Ric, $\operatorname{Ric}^{i}$ denote the curvature tensor and Ricci curvature tensor on $M, M^{i}$, respectively. Moreover, $\nabla^{i} f_{i}$ and $\triangle^{i} f_{i}$ denote gradient and Laplacian of $f_{i}$ on $M_{i}$ and $f_{i}^{\diamond}=f_{i} \triangle^{i} f_{i}+\left(n_{j}-1\right) g_{i}\left(\nabla^{i} f_{i}, \nabla^{i} f_{i}\right), i \neq j$. For the connection and curvatures formulas of a pseudo-Riemannian doubly warped product manifold see, for example, [20, 22].

A vector field $\zeta$ on a (pseudo-)Riemannian manifold $(N, h)$ with metric $h$ is called a conformal vector field with conformal factor $\rho$ if

$$
\mathscr{L}_{\zeta} h=\rho h,
$$

where $\mathscr{L}_{\zeta}$ is the Lie derivative on $N$ with respect to $\zeta$. If $\rho$ is constant or zero, $\zeta$ is called a homothetic or Killing vector field on $N$, respectively. One can redefine conformal vector fields using the following identity. Let $\zeta$ be a vector field on $M$, and then

$$
\left(\mathscr{L}_{\zeta} h\right)(X, Y)=h\left(D_{X} \zeta, Y\right)+h\left(X, D_{Y} \zeta\right)
$$

for any vector fields $X, Y \in \mathfrak{X}(N)$. A vector field $\zeta$ on a manifold $(N, h)$ is called a concurrent vector field if

$$
D_{X} \zeta=X
$$

for any vector field $X \in \mathfrak{X}(N)$ [23]. Let $\zeta$ be a concurrent vector field, and then

$$
\left(\mathscr{L}_{\zeta} h\right)(X, Y)=2 h(X, Y)
$$

and so $\zeta$ is homothetic with factor $\rho=2$. A zero vector field is not concurrent. If both $\zeta$ and $\xi$ are concurrent vector fields, then

$$
D_{X}[\zeta, \xi]=0 .
$$

Also both $\zeta+\xi$ and $\lambda \zeta$ are not concurrent vector fields. Finally, a Killing vector field is not concurrent. For example, a vector field $\alpha \partial_{x}$ is a concurrent vector field on $\left(\mathbb{R}, d x^{2}\right)$ if

$$
D_{\partial_{x}}\left(\alpha \partial_{x}\right)=\partial_{x}
$$


that is, $\alpha=x+a$. Thus concurrent vector fields on $\left(\mathbb{R}, d x^{2}\right)$ are of the form $(x+a) \partial_{x}$.

The following result represents a simple characterization of Killing vector fields, if $(N, h)$ is a pseudo-Riemannian manifold with Riemannian connection $D$. A vector field $\zeta \epsilon$ $\mathfrak{X}(N)$ is a Killing vector field if and only if

$$
h\left(D_{X} \zeta, X\right)=0
$$

for any vector field $X \in \mathfrak{X}(N)$.

The following discussion represents a good tool to characterize Killing vector fields on pseudo-Riemannian warped product manifolds. In $[24,25]$, the authors obtained many characterizations of Killing vector fields on warped product manifolds and on standard static spacetime using these results. Let $M=M_{1} \times_{f} M_{2}$ be a pseudo-Riemannian warped product manifold with warping function $f$. Let $\zeta=\zeta_{1}+\zeta_{2} \epsilon$ $\mathfrak{X}(M)$ be a vector field on $M$. Then

$$
\begin{aligned}
g\left(D_{X} \zeta, X\right)= & g_{1}\left(D_{X_{1}}^{1} \zeta_{1}, X_{1}\right)+f^{2} g_{2}\left(D_{X_{2}}^{2} \zeta_{2}, X_{2}\right) \\
& +f \zeta_{1}(f)\left\|X_{2}\right\|_{2}^{2} \\
\left(\mathscr{L}_{\zeta} g\right)(X, Y)= & \left(\mathscr{L}_{\zeta_{1}}^{1} g_{1}\right)\left(X_{1}, Y_{1}\right) \\
& +f^{2}\left(\mathscr{L}_{\zeta_{2}}^{2} g_{2}\right)\left(X_{2}, Y_{2}\right) \\
& +2 f \zeta_{1}(f) g_{2}\left(X_{2}, Y_{2}\right)
\end{aligned}
$$

for any vector field $X=X_{1}+X_{2} \in \mathfrak{X}(M)$, where $\mathscr{L}_{\zeta_{i}}^{i}$ is the Lie derivative on $M_{i}$ with respect to $\zeta_{i}$, for $i=1,2$.

A pseudo-Riemannian manifold $M$ is said to admit a Ricci curvature collineation if there is a vector field $\zeta \in \mathfrak{X}(M)$ such that

$$
\mathscr{L}_{\zeta} \text { Ric }=0,
$$

where Ric is the Ricci curvature tensor [26]. Finally, spacetime $M$ is said to admit a matter collineation if there is a vector field $\zeta \in \mathfrak{X}(M)$ such that

$$
\mathscr{L}_{\zeta} T=0
$$

where $T$ is the energy-momentum tensor [27]. Einstein's field equation with cosmological constant $\lambda$ is given by

$$
\text { Ric }-\frac{r}{2} g=\kappa T-\lambda g,
$$

where $r$ is the scalar curvature. Suppose that $\zeta$ is a Killing vector field, and then

$$
\mathscr{L}_{\zeta} T=0
$$

that is, $\zeta$ is a matter collineation whereas a matter collineation need not be a Killing vector field. Also, a Killing vector field is a Ricci curvature collineation. The converse is not generally true.

\section{Conformal Vector Fields on Doubly Warped Products}

In this section we investigate the relation between conformal vector fields on doubly warped product manifolds and those conformal vector fields on the product factors. Throughout this section, let $M={ }_{f_{2}} M_{1} \times_{f_{1}} M_{2}$ be a pseudo-Riemannian doubly warped product manifold with the metric tensor $g=f_{2}^{2} g_{1} \oplus f_{1}^{2} g_{2}$ and $f_{i}: M_{i} \rightarrow(0, \infty)$ is a smooth function, where $i=1,2$ and $\left(M_{i}, g_{i}\right)$ are pseudo-Riemannian manifolds. The following result gives us an important identity to study such relation [8].

Proposition 1. Suppose that $\zeta_{1}, X_{1}, Y_{1} \in \mathfrak{X}\left(M_{1}\right)$ and $\zeta_{2}, X_{2}, Y_{2} \in \mathfrak{X}\left(M_{2}\right)$, and then

$$
\begin{aligned}
\left(\mathscr{L}_{\zeta} g\right)(X, Y)= & f_{2}^{2}\left(\mathscr{L}_{\zeta_{1}}^{1} g_{1}\right)\left(X_{1}, \mathrm{Y}_{1}\right) \\
& +f_{1}^{2}\left(\mathscr{L}_{\zeta_{2}}^{2} g_{2}\right)\left(X_{2}, Y_{2}\right) \\
& +2 f_{1} \zeta_{1}\left(f_{1}\right) g_{2}\left(X_{2}, Y_{2}\right) \\
& +2 f_{2} \zeta_{2}\left(f_{2}\right) g_{1}\left(X_{1}, Y_{1}\right),
\end{aligned}
$$

where $\zeta=\zeta_{1}+\zeta_{2}, X=X_{1}+X_{2}$, and $Y=Y_{1}+Y_{2}$ are elements in $\mathfrak{X}(M)$.

In [8], the author considered a characterization of conformal vector fields on doubly warped product manifolds. In fact, it is just a characterization of homothetic vector fields. The following theorem represents a new characterization of conformal vector fields on doubly warped product manifolds but the assumption here is less restrictive.

Theorem 2. A vector field $\zeta=\zeta_{1}+\zeta_{2}$ on a pseudo-Riemannian doubly warped product $M={ }_{f_{2}} M_{1} \times_{f_{1}} M_{2}$ is a conformal vector field with conformal factor $\rho$ if and only if

(1) $\zeta_{i}$ is a conformal vector field on $M_{i}$ with conformal factor $\rho_{i}, i=1,2$,

(2) $\rho_{1}+2 \zeta_{2}\left(\ln f_{2}\right)=\rho_{2}+2 \zeta_{1}\left(\ln f_{1}\right)$.

Moreover, the conformal factor of $\zeta$ is $\rho=\rho_{i}+$ $2 \zeta_{j}\left(\ln f_{j}\right), i \neq j$.

Before proceeding further, one may notice that a doubly warped product metric $g$ on $M$ can be expressed as a conformal metric to a product metric on $M_{1} \times M_{2}$ as follows:

$$
\begin{aligned}
g & =f_{1}^{2} f_{2}^{2}\left(\frac{1}{f_{1}^{2}} g_{1}+\frac{1}{f_{2}^{2}} g_{2}\right)=f_{1}^{2} f_{2}^{2}\left(\bar{g}_{1}+\bar{g}_{2}\right) \\
& =f_{1}^{2} f_{2}^{2} \bar{g} .
\end{aligned}
$$

Let us consider the effect of replacing the metric $g$ on $M$ by $\bar{g}=\bar{g}_{1}+\bar{g}_{2}$. A similar discussion on 4-dimensional spacetime is considered in [26, Chapter 11]. Suppose that $\zeta=\zeta_{1}+\zeta_{2}$ is a conformal vector field on $(M, \bar{g})$ with factor $\bar{\rho}$, and then

$$
\mathscr{L}_{\zeta} g=\left[2 \zeta_{2}\left(\ln f_{2}\right)+2 \zeta_{1}\left(\ln f_{1}\right)+\bar{\rho}\right] g .
$$


Therefore, $\zeta$ is a conformal vector field on $(M, g)$ with factor $\rho=\bar{\rho}+2 \zeta_{2}\left(\ln f_{2}\right)+2 \zeta_{1}\left(\ln f_{1}\right)$. A similar conclusion applies to $\left(M_{i}, g_{i}\right)$ and $\left(M_{i}, \bar{g}_{i}\right)$, where $\bar{\rho}_{i}=\rho-\zeta_{i}\left(\ln f_{i}\right)$. Thus, by using results in [28, Theorem 1], one can easily get the following.

Theorem 3. Let $M={ }_{f_{2}} M_{1} \times{ }_{f_{1}} M_{2}$ be a pseudo-Riemannian doubly warped product equipped with the metric tensor $g=$ $f_{2}^{2} g_{1}+f_{1}^{2} g_{2}$ and let $\bar{g}=\bar{g}_{1}+\bar{g}_{2}$, where $\bar{g}_{i}=\left(1 / f_{i}^{2}\right) g_{i}$. Then,

(1) a Killing vector field $\zeta=\zeta_{i}$ on $\left(M_{i}, \bar{g}_{i}\right)$, for each $i=1,2$, is a Killing vector field on $(M, \bar{g})$,

(2) $(M, \bar{g})$ admits a homothetic vector field if and only if $\left(M_{i}, \bar{g}_{i}\right)$ admits a homothetic vector field for each $i=$ 1,2 ,

(3) each conformal vector field on $(M, \bar{g})$ is a conformal vector field on $(M, g)$.

The above theorem together with Theorem 2 implies the following results.

Theorem 4. Let $\zeta=\zeta_{1}+\zeta_{2}$ be a vector field on a pseudo-Riemannian doubly warped product $M={ }_{f_{2}} M_{1} \times_{f_{1}} M_{2}$ equipped with the metric tensor $g=f_{2}^{2} g_{1}+f_{1}^{2} g_{2}$. Assume that $\zeta_{i}$ is a Killing vector field on $\left(M_{i}, g_{i}\right)$ for each $i=1,2$ and $\zeta_{1}\left(\ln f_{1}\right)=\zeta_{2}\left(\ln f_{2}\right)$. Then $\zeta$ is a conformal vector field on $M$.

Theorem 5. Let $\zeta_{i} \in \mathfrak{X}\left(M_{i}\right)$ be homothetic vector fields on $\left(M_{i}, g_{i}\right)$ with factors $a_{i}$ for each $i=1,2$. Assume that $\zeta_{1}\left(f_{1}\right)=$ $\zeta_{2}\left(f_{2}\right)=0$. Then, $\zeta=a_{2} \zeta_{1}+a_{1} \zeta_{2}$ is a homothetic vector field on $(M, g)$ with factor $a_{1} a_{2}$.

Corollary 6. The dimension of the conformal group $C(M, g)$ on a pseudo-Riemannian doubly warped product $M=f_{f_{2}}$ $M_{1} \times_{f_{1}} M_{2}$ is at least

$$
\operatorname{dim} K_{1}\left(M_{1}, \bar{g}_{1}\right)+\operatorname{dim} K_{1}\left(M_{2}, \bar{g}_{2}\right),
$$

where $K_{i}\left(M_{i}, \bar{g}_{i}\right)$ is the isometry group of $\left(M_{i}, \bar{g}_{i}\right)$.

Again Theorem 2 together with Lemma 2.1 in [16] yields the following result.

Theorem 7. Let $\zeta=\zeta_{1}+\zeta_{2}$ be a vector field on a pseudoRiemannian doubly warped product $M={ }_{f_{2}} M_{1} \times{ }_{f_{1}} M_{2}$ such that

(1) $\zeta_{i}$ is a conformal vector field on $M_{i}$ with conformal factor $\rho_{i}, i=1,2$,

(2) $\rho_{1}+2 \zeta_{2}\left(\ln f_{2}\right)=\rho_{2}+2 \zeta_{1}\left(\ln f_{1}\right)$.

Then, $\zeta$ preserves the Ricci curvature if and only if $H^{u}=0$, where $u=\rho_{2}+2 \zeta_{1}\left(\ln f_{1}\right)$. Moreover, $\zeta$ preserves the conformal class of the Ricci tensor (i.e., $\mathscr{L}_{\zeta}$ Ric $=\lambda g$ for some function $\lambda$ ) if and only if $\nabla(\operatorname{div}(\zeta))$ is a conformal vector field.

Theorem 8. Let $\zeta=\zeta_{1}+\zeta_{2}$ be a vector field on a pseudoRiemannian doubly warped product $M={ }_{f_{2}} M_{1} \times_{f_{1}} M_{2} . \zeta$ has constant length along the integral curve $\alpha$ of the vector field $X=$ $X_{1}+X_{2} \in \mathfrak{X}(M)$ if one of the following conditions holds:

(1) $X_{i}\left(f_{i}\right)=0$ and $\zeta_{i}$ is parallel along $\pi_{i} \circ \alpha$ for each $i=1,2$.
(2) $X_{i}\left(f_{i}\right)=0$ and $\zeta_{i}$ has a constant length along $\pi_{i} \circ \alpha$ for each $i=1,2$.

Proof. Let $\zeta=\zeta_{1}+\zeta_{2} \in \mathfrak{X}(M)$ be a vector field on $M$. Then,

$$
\begin{aligned}
g\left(D_{X} \zeta, \zeta\right)= & g\left(D_{X_{1}+X_{2}} \zeta_{1}+\zeta_{2}, \zeta\right) \\
= & g\left(D_{X_{1}} \zeta_{1}+D_{X_{1}} \zeta_{2}+D_{X_{2}} \zeta_{1}+D_{X_{2}} \zeta_{2}, \zeta\right) \\
= & f_{2}^{2} g_{1}\left(D_{X_{1}}^{1} \zeta_{1}, \zeta_{1}\right)+f_{1}^{2} g_{2}\left(D_{X_{2}}^{2} \zeta_{2}, \zeta_{2}\right) \\
& +f_{1} X_{1}\left(f_{1}\right)\left\|\zeta_{2}\right\|_{2}^{2}+f_{2} X_{2}\left(f_{2}\right)\left\|\zeta_{1}\right\|_{1}^{2}
\end{aligned}
$$

In both cases $X_{i}\left(f_{i}\right)=0$, and hence

$$
g\left(D_{X} \zeta, \zeta\right)=f_{2}^{2} g_{1}\left(D_{X_{1}}^{1} \zeta_{1}, \zeta_{1}\right)+f_{1}^{2} g_{2}\left(D_{X_{2}}^{2} \zeta_{2}, \zeta_{2}\right)
$$

The first condition implies that $D_{X_{i}}^{i} \zeta_{i}=0$ and so $g_{i}\left(D_{X_{i}}^{i} \zeta_{i}\right.$, $\left.\zeta_{i}\right)=0$. The second condition implies that $g_{i}\left(\zeta_{i}, \zeta_{i}\right)$ is constant and so

$$
0=2 g_{i}\left(D_{X_{i}}^{i} \zeta_{i}, \zeta_{i}\right)
$$

and therefore

$$
g\left(D_{X} \zeta, \zeta\right)=0
$$

that is, $\zeta$ has a constant length along the integral curve $\alpha$ of the vector field $X$

Theorem 9. Let $\zeta=\zeta_{1}+\zeta_{2} \in \mathfrak{X}(M)$ be a conformal vector field on a pseudo-Riemannian doubly warped product $M=f_{f_{2}}$ $M_{1} \times_{f_{1}} M_{2}$ along a curve $\alpha$ with unit tangent vector $T=V_{1}+$ $V_{2}$. Then,

$$
\begin{aligned}
& \operatorname{div}(\zeta)=n\left[f_{2}^{2} g_{1}\left(D_{V_{1}}^{1} \zeta_{1}, V_{1}\right)+f_{1}^{2} g_{2}\left(D_{V_{2}}^{2} \zeta_{2}, V_{2}\right)\right. \\
& \left.\quad+f_{2} \zeta_{2}\left(f_{2}\right)\left\|V_{1}\right\|_{1}^{2}+f_{1} \zeta_{1}\left(f_{1}\right)\left\|V_{2}\right\|_{2}^{2}\right] .
\end{aligned}
$$

Proof. Let $\zeta$ be a conformal vector field with conformal factor $\rho$. Then,

$$
\left(\mathscr{L}_{\zeta} g\right)(X, Y)=\rho g(X, Y) .
$$

Let $X=Y=T$, then

$$
2 g\left(D_{T} \zeta, T\right)=\rho g(T, T),
$$

and then the conformal factor $\rho$ is given by

$$
\rho=2 g\left(D_{T} \zeta, T\right) \text {. }
$$

Suppose that $\zeta=\zeta_{1}+\zeta_{2}$ and $T=V_{1}+V_{2}$, and then

$$
\begin{aligned}
\rho= & 2 g\left(D_{V_{1}} \zeta_{1}+D_{V_{1}} \zeta_{2}+D_{V_{2}} \zeta_{1}+D_{V_{2}} \zeta_{2}, T\right) \\
= & 2 f_{2}^{2} g_{1}\left(D_{V_{1}}^{1} \zeta_{1}, V_{1}\right)+2 f_{1}^{2} g_{2}\left(D_{V_{2}}^{2} \zeta_{2}, V_{2}\right) \\
& +2 f_{2} \zeta_{2}\left(f_{2}\right)\left\|V_{1}\right\|_{1}^{2}+2 f_{1} \zeta_{1}\left(f_{1}\right)\left\|V_{2}\right\|_{2}^{2} .
\end{aligned}
$$

But the conformal factor is given by

$$
2 \operatorname{div}(\zeta)=\rho n
$$

which completes the proof. 
3.1. Conformal Vector Fields on Doubly Warped Spacetime. Doubly warped spacetime is a doubly warped product manifold $M={ }_{f_{2}} M_{1} \times{ }_{f_{1}} M_{2}$, where one of the factors, say $M_{1}$, has a Lorentz signature and the second is Riemannian. Ramos et al. considered an invariant characterization of 4-dimensional doubly warped spacetime [21]. Among many other results, they obtained necessary and sufficient conditions for (locally) double warped spacetime to be conformally related to $1+3$ or $2+2$ decomposable spacetime. Then they studied the conformal algebra of $2+2$ decomposable spacetime in section IV and $1+3$ decomposable spacetime in section V. For a detailed discussion of conformally related $1+3$ and $2+2$ reducible spacetime see $[29,30]$ and, for an extensive selfcontained study of conformal symmetry of 4-dimensional spacetime, the reader is referred to [26].

We restrict our study of conformal vector fields on doubly warped spacetime to $\operatorname{dim}\left(M_{1}\right) \leq 2$ since this case generalizes some well-known exact solutions for the Einstein field equations and the beginning of this section represents such study irrespective of the dimension of the factors. For this case, either $\operatorname{dim}\left(M_{1}\right)=1$ or $\operatorname{dim}\left(M_{1}\right)=2$. In the following we deal with both subcases separately. Let us first consider doubly warped spacetime with a 1-dimensional base.

Let $(M, g)$ be a Riemannian manifold and $I$ be an open connected interval equipped with the metric $-d t^{2}$. Doubly warped spacetime $\bar{M}={ }_{f} I \times{ }_{\sigma} M$ is the product $I \times M$ furnished with the metric

$$
\bar{g}=-f^{2} d t^{2} \oplus \sigma^{2} g
$$

where $f: M \rightarrow(0, \infty)$ and $\sigma: I \rightarrow(0, \infty)$ are smooth functions. $\bar{M}$ is generalized Robertson-Walker spacetime if $f$ is constant and standard static spacetime if $\sigma$ is constant.

An investigation of 4 -dimensional spacetime that is conformally related to $1+3$ reducible spacetime was carried out with many examples in the aforementioned references $[21,30]$. A classification of this spacetime according to its conformal algebra is considered in the first reference whereas a special attention is paid to gradient conformal vector fields in the second reference.

Theorem 10. A time-like vector field $\bar{\zeta}=h \partial_{t}$ is a conformal vector field on doubly warped spacetime $\bar{M}={ }_{f} I \times{ }_{\sigma} M$ if and only if $h=a \sigma$, where $a$ is a nonnegative constant. Moreover, the conformal factor is $\rho=2 \dot{h}$.

Proof. If $h=0$, then $a=0$ and the result is obvious. Now, we assume that $h \neq 0$. Using (16), we get that

$$
\begin{aligned}
\left(\overline{\mathscr{L}}_{\bar{\zeta}} \bar{g}\right)(\bar{X}, \bar{Y})= & -2 x y f^{2}[\dot{h}+\zeta(\ln f)] \\
& +\sigma^{2}\left(\mathscr{L}_{\zeta} g\right)(X, Y)+2 h \sigma \dot{\sigma} g(X, Y) \\
= & -2 x y f^{2} \dot{h}+2 h \sigma \dot{\sigma} g(X, Y) \\
= & 2 \dot{h} f^{2} g_{I}\left(x \partial_{t}, y \partial_{t}\right)+\frac{2 h \dot{\sigma}}{\sigma} \sigma^{2} g(X, Y) .
\end{aligned}
$$

Suppose that $h=a \sigma$, and then

$$
(\overline{\mathscr{L}} \bar{\zeta} \bar{g})(\bar{X}, \bar{Y})=2 \dot{h} \bar{g}(\bar{X}, \bar{Y})
$$

that is, $\bar{\zeta}=h \partial_{t}$ is a conformal vector field with conformal factor $\rho=2 \dot{h}$. Conversely, suppose that $\bar{\zeta}=h \partial_{t} \in \mathfrak{X}(\bar{M})$ is a conformal vector field with factor $\rho$, and then

$$
\left(\overline{\mathscr{L}}_{\bar{\zeta}} \bar{g}\right)(\bar{X}, \bar{Y})=\rho \bar{g}(\bar{X}, \bar{Y})
$$

for any vector fields $\bar{X}, \bar{Y} \in \mathfrak{X}(\bar{M})$. Now, by (16), we get that

$$
\rho \bar{g}(\bar{X}, \bar{Y})=2 \dot{h} f^{2} g_{I}\left(x \partial_{t}, y \partial_{t}\right)+\frac{2 h \dot{\sigma}}{\sigma} \sigma^{2} g(X, Y) \text {. }
$$

Let $X=Y=0$, and we get that

$$
\rho \bar{g}\left(x \partial_{t}, y \partial_{t}\right)=2 \dot{h} f^{2} g_{I}\left(x \partial_{t}, y \partial_{t}\right) ;
$$

that is, $\rho=2 \dot{h}$. Now, let $x=y=0$, and we get that $\rho \sigma=2 h \dot{\sigma}$.

These two differential equations imply that $h=a \sigma$ for some positive constant $a$.

Theorem 11. A vector field $\bar{\zeta}=h \partial_{t}+\zeta$ is a Killing vector field on doubly warped spacetime $\bar{M}={ }_{f} I \times{ }_{\sigma} M$ if and only if one of the following conditions holds:

(1) $\bar{\zeta}$ is time-like and $\dot{h}=\dot{\sigma}=0$.

(2) $\bar{\zeta}$ is space-like where $\zeta$ is a Killing vector field on $M$ and $\zeta(f)=0$.

(3) $\dot{h}=-\zeta(\ln f)$ and $\zeta$ is a conformal vector field on $M$ with conformal factor $\rho_{2}=-2 h \dot{\sigma} / \sigma$.

Proof. The first assertion is a special case of the above theorem. For the second assertion, let $h=0$ in (16). Thus,

$$
\left(\overline{\mathscr{L}}_{\bar{\zeta}} \bar{g}\right)(\bar{X}, \bar{Y})=-2 x y f \zeta(f)+\sigma^{2}\left(\mathscr{L}_{\zeta} g\right)(X, Y) .
$$

Suppose that $\zeta$ is a Killing vector field on $M$ and $\zeta(f)=0$, and then

$$
\left(\overline{\mathscr{L}}_{\bar{\zeta}} \bar{g}\right)(\bar{X}, \bar{Y})=0
$$

The converse is direct. Finally, let $\bar{\zeta}$ be a Killing vector field on $\bar{M}={ }_{f} I \times{ }_{\sigma} M$, and then

$$
\begin{aligned}
0= & \left(\overline{\mathscr{L}}_{\bar{\zeta}} \bar{g}\right)(\bar{X}, \bar{Y}) \\
0= & -2 x y f^{2}[\dot{h}+\zeta(\ln f)]+\sigma^{2}\left(\mathscr{L}_{\zeta} g\right)(X, Y) \\
& +2 h \sigma \dot{\sigma} g(X, Y) .
\end{aligned}
$$

Let $X=Y=0$, and then

$$
-2 x y f^{2}[\dot{h}+\zeta(\ln f)]=0
$$

and so $\dot{h}=-\zeta(\ln f)$. Thus,

$$
\left(\mathscr{L}_{h \partial_{t}}^{I} g_{I}\right)\left(x \partial_{t}, y \partial_{t}\right)=-2 \zeta(\ln f) g_{I}\left(x \partial_{t}, y \partial_{t}\right) ;
$$


that is, $h \partial_{t}$ is a conformal vector field on $I$ with conformal factor $-2 \zeta(\ln f)$. Now let $x=y=0$, and then

$$
\left(\mathscr{L}_{\zeta} g\right)(X, Y)=-\frac{2 h \dot{\sigma}}{\sigma} g(X, Y)
$$

that is, $\zeta$ is a conformal vector field on $M$ with conformal factor $\rho=-2 h \dot{\sigma} / \sigma$.

Conversely, suppose that $\dot{h}=-\zeta(\ln f)$ and $\zeta$ is a conformal vector field on $M$ with conformal factor $\rho=-2 h \dot{\sigma} /$ $\sigma$, and then

$$
\left(\mathscr{L}_{\zeta} g\right)(X, Y)=-\frac{2 h \dot{\sigma}}{\sigma} g(X, Y)
$$

Thus,

$$
\begin{aligned}
\left(\overline{\mathscr{L}}_{\bar{\zeta}} \bar{g}\right)(\bar{X}, \bar{Y})= & -2 x y f^{2}[\dot{h}+\zeta(\ln f)] \\
& +\sigma^{2}\left(\mathscr{L}_{\zeta} g\right)(X, Y)+2 h \sigma \dot{\sigma} g(X, Y) \\
= & 0 ;
\end{aligned}
$$

that is, $\bar{\zeta}=h \partial_{t}+\zeta$ is a Killing vector field on $\bar{M}={ }_{f} I \times{ }_{\sigma} M$.

Corollary 12. Let $\bar{\zeta}=h \partial_{t}+\zeta$ be vector field on doubly warped spacetime $\bar{M}={ }_{f} I \times{ }_{\sigma} M$ obeying Einstein's field equation. Then,

(1) $\bar{M}$ admits a time-like matter collineation $\bar{\zeta}=h \partial_{t}$ if $\dot{h}=$ $\dot{\sigma}=0$,

(2) $\bar{M}$ admits a space-like matter collineation $\bar{\zeta}=\zeta$ if $\zeta$ is a Killing vector field on $M$ and $\zeta(f)=0$,

(3) $\bar{M}$ admits a matter collineation $\bar{\zeta}=h \partial_{t}+\zeta$ if $\dot{h}=$ $-\zeta(\ln f)$ and $\zeta$ is a conformal vector field on $M$ with conformal factor $\rho_{2}=-2 h \dot{\sigma} / \sigma$.

The following result is a direct consequence of Theorem 9.

Corollary 13. Let $\bar{\zeta}=h \partial_{t}+\zeta \in \mathfrak{X}(\bar{M})$ be a conformal vector field on doubly warped spacetime $\bar{M}={ }_{f} I \times{ }_{\sigma} M$ along a curve $\alpha$ with unit tangent vector $\bar{V}=v \partial_{t}+V$. Then the conformal factor $\rho$ of $\bar{\zeta}$ is given by

$$
\rho=2 \dot{h}+2 \sigma^{2} g([\zeta, V], V)+2\left(h \sigma \dot{\sigma}-\dot{h} \sigma^{2}\right) g(V, V) .
$$

In the sequel, we present doubly warped spacetime with a 2 -dimensional base. In this subcase, $2+n$ doubly warped spacetime is considered to be doubly warped product manifold with a 2-dimensional pseudo-Riemannian base and $n$-dimensional Riemannian fibre; $2+n$ doubly warped spacetime is clearly conformal to a product manifold. In $[21,29]$ and references therein, the Lie conformal algebra of conformally related $2+2$ reducible spacetime is extensively studied. Many interesting results and examples are given there. For example, in [29], Carot and Tupper considered an invariant characterization that imposes conditions on the conformal factor and on two null vectors. Moreover, Van den Bergh considered non-conformally flat perfect fluids spacetime which is conformally $2+2$ decomposable spacetime with factor spaces of constant curvature [31].

It is well-known that each 2-dimensional manifold is conformally flat. Thus we may simply take the base manifold as $\left(\mathbb{R}^{2}, d s^{2}\right)$, where $d s^{2}=-d t^{2}+d x^{2}$. Let $\bar{M}={ }_{f} \mathbb{R}^{2} \times{ }_{\sigma} M$ be $(2+n)$-dimensional doubly warped product spacetime furnished with the metric $\bar{g}=f^{2} d s^{2}+\sigma^{2} g$.

Proposition 14. Suppose that $h(t) \partial_{t}+u(x) \partial_{x}, h_{i}(t) \partial_{t}+$ $u_{i}(x) \partial_{x} \in \mathfrak{X}\left(\mathbb{R}^{2}\right), i=1,2$, and $\zeta, X_{1}, X_{2} \in \mathfrak{X}(M)$, and then

$$
\begin{aligned}
\left(\overline{\mathscr{L}}_{\bar{\zeta}} \bar{g}\right)(\bar{X}, \bar{Y})= & 2 f^{2}\left(-h_{1} h_{2} \dot{h}+u_{1} u_{2} u^{\prime}\right) \\
& +\sigma^{2}\left(\mathscr{L}_{\zeta} g\right)(X, Y) \\
& +2 \sigma\left(h \sigma_{t}+u \sigma_{x}\right) g(X, Y) \\
& +2 f \zeta(f)\left(-h_{1} h_{2}+u_{1} u_{2}\right),
\end{aligned}
$$

where $\bar{\zeta}=h \partial_{t}+u \partial_{x}+\zeta$ and $\bar{X}_{i}=h_{i} \partial_{t}+u_{i} \partial_{x}+X_{i}$ are elements in $\mathfrak{X}(\bar{M})$.

Corollary 15. A vector field $\bar{\zeta}=h(t) \partial_{t}+u(x) \partial_{x}+\zeta \in \mathfrak{X}(\bar{M})$ is a Killing vector field if one of the following conditions holds:

(1) $\zeta=0, h=a, u=b$, and $a \sigma_{t}+b \sigma_{x}=0$.

(2) $\zeta$ is a Killing vector field on $M, h=u=0$, and $\zeta(f)=0$.

3.2. Concurrent Vector Fields on Doubly Warped Spacetime. In this subsection, we study concurrent vector fields on doubly warped spacetime with a 1-dimensional base. One can extend most of the results to doubly warped spacetime with a 2-dimensional base. Throughout this subsection, let $\bar{M}={ }_{f} I \times{ }_{\sigma} M$ be doubly warped spacetime equipped with the metric tensor $\bar{g}=-f^{2} d t^{2} \oplus \sigma^{2} g$.

Theorem 16. A vector field $\bar{\zeta}=h \partial_{t}+\zeta$ on doubly warped spacetime $\bar{M}={ }_{f} I \times{ }_{\sigma} M$ is a concurrent vector field if

(1) $\zeta$ and $h \partial_{t}$ are concurrent vector fields on $M$ and $I$, respectively,

(2) both $f$ and $\sigma$ are constant.

Proof. Suppose that $\bar{X}=x \partial_{t}+X \in \mathfrak{X}(\bar{M})$ is any vector field on $\bar{M}$, and then

$$
\begin{aligned}
\bar{D} \bar{X}_{\bar{\zeta}}= & \bar{D}_{x \partial_{t}} h \partial_{t}+\bar{D}_{x \partial_{t}} \zeta+\bar{D}_{X} h \partial_{t}+\bar{D}_{X} \zeta \\
= & x \dot{h} \partial_{t}+\frac{x h f}{\sigma^{2}} \nabla f+x(\ln \sigma) \zeta+\zeta(\ln f) x \partial_{t} \\
& +h\left(\ln \sigma \dot{)} X+X(\ln f) h \partial_{t}+D_{X} \zeta\right. \\
& -\frac{\sigma \dot{\sigma}}{f^{2}} g(X, \zeta) \partial_{t} \\
= & {\left[x \dot{h}+\zeta(\ln f) x+X(\ln f) h-\frac{\sigma \dot{\sigma}}{f^{2}} g(X, \zeta)\right] \partial_{t} } \\
& +\frac{x h f}{\sigma^{2}} \nabla f+x(\ln \sigma) \zeta+h(\ln \sigma) X+D_{X} \zeta .
\end{aligned}
$$


TABLE 1

\begin{tabular}{lcccc}
\hline & Case & & $\zeta$ & $\sigma$ \\
\hline$h=0$ & $\dot{\sigma}=0$ & $\zeta=(x+a) \partial_{x}$ & Constant & $r(x+a)$ \\
$k=0$ & $f^{\prime}=0$ & $\zeta=(t+a) \partial_{t}$ & $r(t+a)$ & Constant \\
$f^{\prime}=0$ & $\dot{\sigma}=0$ & $\zeta=(t+a) \partial_{t}+(x+a) \partial_{x}$ & Constant & Constant \\
\hline
\end{tabular}

Now suppose that $h \partial_{t}$ and $\zeta$ are concurrent vector fields; then $\dot{h}=1$ and $D_{X} \zeta=X$. If both $\sigma$ and $f$ are constant, then

$$
\bar{D}_{\bar{X}} \bar{\zeta}=x \dot{h} \partial_{t}+D_{X} \zeta=\bar{X}
$$

that is, $\bar{\zeta}$ is concurrent.

It is well-known that a homothetic vector field is a matter collineation. Thus the above theorem yields the following result.

Corollary 17. A vector field $\bar{\zeta}=h \partial_{t}+\zeta$ on doubly warped spacetime $\bar{M}={ }_{f} I \times{ }_{\sigma} M$ is a matter collineation if

(1) $\zeta$ and $h \partial_{t}$ are concurrent vector fields on $M$ and $I$, respectively,

(2) both $f$ and $\sigma$ are constant.

Theorem 18. Let $\bar{\zeta}=h \partial_{t}+\zeta$ be a concurrent vector field on doubly warped spacetime $\bar{M}={ }_{f} I \times{ }_{\sigma} M$ equipped with the metric tensor $\bar{g}=-f^{2} d t^{2} \oplus \sigma^{2} g$. Then, $\zeta$ is a concurrent vector field on $M$ if one of the following conditions holds:

(1) $h=0$, or

(2) $\sigma$ is a constant; that is, $\bar{M}$ is standard static spacetime.

Moreover, condition (1) implies condition (2) and the converse is true if $f$ is not constant.

Proof. From the above proof we have

$$
\begin{aligned}
& x=x \dot{h}+\zeta(\ln f) x+X(\ln f) h-\frac{\sigma \dot{\sigma}}{f^{2}} g(X, \zeta), \\
& X=\frac{x h f}{\sigma^{2}} \nabla f+x(\ln \sigma) \zeta+h(\ln \sigma) X+D_{X} \zeta
\end{aligned}
$$

for any $x$ and $X$. Let $x=0$, and then

$$
\begin{aligned}
0 & =X(\ln f) h-\frac{\sigma \dot{\sigma}}{f^{2}} g(X, \zeta), \\
X & =h(\ln \sigma) X+D_{X} \zeta \\
D_{X} \zeta & =\left[1-\frac{h \dot{\sigma}}{\sigma}\right] X .
\end{aligned}
$$

Thus $\zeta$ is concurrent if $h \dot{\sigma}=0$.

If $h=0$, then

$$
\frac{\sigma \dot{\sigma}}{f^{2}} g(X, \zeta)=0
$$

If $g(X, \zeta)=0$ then $\bar{\zeta}=0$ which is a contradiction and so $\dot{\sigma}=0$; that is, $\bar{M}$ is standard static spacetime.

If $\dot{\sigma}=0$, then

$$
X(f) h=0
$$

which implies that $h=0$ for a nonconstant function $f$.

Theorem 19. Let $\bar{\zeta}=h \partial_{t}+\zeta$ be a concurrent vector field on $\bar{M}$, where $h \neq 0$. Then $\zeta$ and $h \partial_{t}$ are concurrent vector fields on $M$ and $I$, respectively, if $\dot{\sigma}=0$. In this case $f$ is also constant.

Example 20. Table 1 summarizes all three cases of concurrent vector fields on the 2-dimensional doubly warped spacetime of the form $\bar{M}={ }_{f} I \times{ }_{\sigma} \mathbb{R}$ equipped with the metric $\bar{g}=$ $-f^{2} d t^{2} \oplus \sigma^{2} d x^{2}$. For more details see Appendix.

\section{Ricci Soliton on Doubly Warped Spacetime}

A smooth vector field $\zeta$ on a Riemannian manifold $(M, g)$ is said to define a Ricci soliton if

$$
\frac{1}{2}\left(\mathscr{L}_{\zeta} g\right)(X, Y)+\operatorname{Ric}(X, Y)=\lambda g(X, Y)
$$

where $\mathscr{L}_{\zeta}$ denotes the Lie derivative of the metric tensor $g$, Ric is the Ricci curvature, and $\lambda$ is a constant [32-36].

Theorem 21. Let $(\bar{M}, \bar{g}, \bar{\zeta}, \lambda)$ be a Ricci soliton where $\bar{M}={ }_{f}$ $I \times_{\sigma} M$ is doubly warped spacetime and $\bar{\zeta}=h \partial_{t}+\zeta \in \mathfrak{X}(\bar{M})$. Then

$$
\begin{aligned}
& \dot{h}=\frac{1}{f^{2}}\left(\lambda f^{2}-f \zeta(f)+\frac{n}{\sigma} \ddot{\sigma}+\frac{f^{\diamond}}{\sigma^{2}}\right), \\
& \frac{1}{2} \sigma^{2}\left(\mathscr{L}_{\zeta} g\right)(X, Y)+\operatorname{Ric}(X, Y)-\frac{1}{f} H^{f}(X, Y) \\
&=\left(\lambda \sigma^{2}-h \sigma \dot{\sigma}+\frac{\sigma^{\diamond}}{f^{2}}\right) g(X, Y) .
\end{aligned}
$$

Proof. Let $\bar{M}={ }_{f} I \times{ }_{\sigma} M$ be a Ricci soliton, and then

$$
\frac{1}{2}\left(\overline{\mathscr{L}}_{\bar{\zeta}} \bar{g}\right)(\bar{X}, \bar{Y})+\overline{\operatorname{Ric}}(\bar{X}, \bar{Y})=\lambda \bar{g}(\bar{X}, \bar{Y}),
$$

where $\bar{X}=x \partial_{t}+X$ and $\bar{Y}=y \partial_{t}+Y$ are vector fields on $\bar{M}$. Then

$$
\begin{gathered}
-\lambda x y f^{2}+\lambda \sigma^{2} g(X, Y)=\frac{1}{2}\left(\overline{\mathscr{L}}_{\bar{\zeta}} \bar{g}\right)(\bar{X}, \bar{Y}) \\
+\overline{\operatorname{Ric}}(\bar{X}, \bar{Y})=\frac{1}{2}\left[-2 x y f^{2}[\dot{h}+\zeta(\ln f)]\right. \\
\left.+\sigma^{2}\left(\mathscr{L}_{\zeta} g\right)(X, Y)+2 h \sigma \dot{\sigma} g(X, Y)\right]
\end{gathered}
$$




$$
\begin{aligned}
+ & \overline{\operatorname{Ric}}\left(x \partial_{t}, y \partial_{t}\right)+\overline{\operatorname{Ric}}\left(x \partial_{t}, Y\right)+\overline{\operatorname{Ric}}\left(X, y \partial_{t}\right) \\
+ & \overline{\operatorname{Ric}}(X, Y) \\
- & \lambda x y f^{2}+\lambda \sigma^{2} g(X, Y)=\frac{1}{2}\left[-2 x y f^{2}[\dot{h}+\zeta(\ln f)]\right. \\
+ & \left.\sigma^{2}\left(\mathscr{L}_{\zeta} g\right)(X, Y)+2 h \sigma \dot{\sigma} g(X, Y)\right]+(n-1) \\
& \cdot\left(\frac{x \dot{\sigma}}{\sigma}\right) Y(\ln f)+(n-1)\left(\frac{y \dot{\sigma}}{\sigma}\right) X(\ln f)+\frac{n}{\sigma} x y \ddot{\sigma} \\
& +x y \frac{f^{\diamond}}{\sigma^{2}}+\operatorname{Ric}(X, Y)-\frac{1}{f} H^{f}(X, Y)-\frac{\sigma^{\diamond}}{f^{2}} g(X, Y) .
\end{aligned}
$$

Let $X=Y=0$, and we get

$$
\begin{array}{r}
x y f^{2}[\dot{h}+\zeta(\ln f)]-\frac{n}{\sigma} x y \ddot{\sigma}-x y \frac{f^{\diamond}}{\sigma^{2}}-\lambda f^{2} x y=0 \\
x y\left[\dot{h} f^{2}+f \zeta(f)-\frac{n}{\sigma} \ddot{\sigma}-\frac{f^{\diamond}}{\sigma^{2}}-\lambda f^{2}\right]=0
\end{array}
$$

and so

$$
\begin{aligned}
\dot{h} f^{2} & =\lambda f^{2}-f \zeta(f)+\frac{n}{\sigma} \ddot{\sigma}+\frac{f^{\diamond}}{\sigma^{2}} \\
\dot{h} & =\frac{1}{f^{2}}\left(\lambda f^{2}-f \zeta(f)+\frac{n}{\sigma} \ddot{\sigma}+\frac{f^{\diamond}}{\sigma^{2}}\right) .
\end{aligned}
$$

Now, let us put $x=y=0$, and then

$$
\begin{aligned}
\lambda \sigma^{2} g(X, Y)= & \frac{1}{2}\left[\sigma^{2}\left(\mathscr{L}_{\zeta} g\right)(X, Y)+2 h \sigma \dot{\sigma} g(X, Y)\right] \\
& +\operatorname{Ric}(X, Y)-\frac{1}{f} H^{f}(X, Y) \\
& -\frac{\sigma^{\diamond}}{f^{2}} g(X, Y)
\end{aligned}
$$

and so

$$
\begin{gathered}
\frac{1}{2} \sigma^{2}\left(\mathscr{L}_{\zeta} g\right)(X, Y)+\operatorname{Ric}(X, Y)-\frac{1}{f} H^{f}(X, Y) \\
=\left(\lambda \sigma^{2}-h \sigma \dot{\sigma}+\frac{\sigma^{\diamond}}{f^{2}}\right) g(X, Y) .
\end{gathered}
$$

The following corollaries are consequences of the above theorem.

Corollary 22. Let $(\bar{M}, \bar{g}, \bar{\zeta}, \lambda)$ be a Ricci soliton where $\bar{M}={ }_{f}$ $I \times{ }_{\sigma} M$ is doubly warped spacetime and $\bar{\zeta}=h \partial_{t}+\zeta \in \mathfrak{X}(\bar{M})$. Then

(1) $h \partial_{t}$ is a conformal vector field on I with factor $\left(2 / f^{2}\right)\left(\lambda f^{2}-f \zeta(f)+(n / \sigma) \ddot{\sigma}+f^{\diamond} / \sigma^{2}\right)$,
(2) $(M, g, \zeta, \lambda)$ is a Ricci soliton if $f=\sigma=1$,

(3) $(M, g, \zeta, \lambda)$ is a Ricci soliton if $\sigma=1$ and $H^{f}=0$.

Theorem 23. Let $(\bar{M}, \bar{g}, \bar{\zeta}, \lambda)$ be a Ricci soliton where $\bar{M}={ }_{f} I \times{ }_{\sigma} M$ is doubly warped spacetime and $\bar{\zeta}=h \partial_{t}+\zeta \epsilon$ $\mathfrak{X}(\bar{M})$ is a conformal vector field on $\bar{M}$ with factor $2 \rho$. Then $(M, g)$ is Einstein manifold with factor $\mu=(\lambda-\rho) \sigma^{2}+\sigma^{\diamond} / f^{2}$ if $f$ is constant.

Proof. Let $(\bar{M}, \bar{g}, \bar{\zeta}, \bar{\lambda})$ be a Ricci soliton where $\bar{M}={ }_{f} I \times{ }_{\sigma} M$ is doubly warped spacetime and $\bar{\zeta}=h \partial_{t}+\zeta \in \mathfrak{X}(\bar{M})$ is a conformal vector field on $\bar{M}$. Then,

$$
\overline{\operatorname{Ric}}(\bar{X}, \bar{Y})=(\lambda-\rho) \bar{g}(\bar{X}, \bar{Y}) .
$$

Let $x=y=0$, and then

$$
\overline{\operatorname{Ric}}(X, Y)=(\lambda-\rho) \sigma^{2} g(X, Y) .
$$

This equation implies that

$$
\begin{aligned}
\operatorname{Ric} & (X, Y)-\frac{1}{f} H^{f}(X, Y)-\frac{\sigma^{\diamond}}{f^{2}} g(X, Y) \\
= & (\lambda-\rho) \sigma^{2} g(X, Y)
\end{aligned}
$$

and so

$$
\begin{aligned}
\operatorname{Ric} & (X, Y)-\frac{1}{f} H^{f}(X, Y) \\
= & {\left[(\lambda-\rho) \sigma^{2}+\frac{\sigma^{\diamond}}{f^{2}}\right] g(X, Y) . }
\end{aligned}
$$

Corollary 24. Let $(\bar{M}, \bar{g}, \bar{\zeta}, \lambda)$ be a Ricci soliton where $\bar{M}={ }_{f} I \times{ }_{\sigma} M$ is doubly warped spacetime and $\bar{\zeta}=h \partial_{t}+\zeta \epsilon$ $\mathfrak{X}(\bar{M})$ is a homothetic vector field on $\bar{M}$ with factor $2 c$. Then,

$$
\lambda=c-\frac{1}{f^{2}}\left(\frac{n}{\sigma} \ddot{\sigma}+\frac{f^{\diamond}}{\sigma^{2}}\right)
$$

Proof. Let $(\bar{M}, \bar{g}, \bar{\zeta}, \lambda)$ be a Ricci soliton and $\bar{\zeta}=h \partial_{t}+\zeta \epsilon$ $\mathfrak{X}(\bar{M})$ be a homothetic vector field on $\bar{M}$, and then

$$
(\lambda-c) \bar{g}(\bar{X}, \bar{Y})=\overline{\operatorname{Ric}}(\bar{X}, \bar{Y})
$$

for any vector fields $\bar{X}=x \partial_{t}+X$ and $\bar{Y}=y \partial_{t}+Y$. Let us take $X=Y=0$, and then

$$
\begin{aligned}
\overline{\operatorname{Ric}}\left(x \partial_{t}, y \partial_{t}\right) & =(\lambda-c) \bar{g}\left(x \partial_{t}, y \partial_{t}\right) \\
x y \frac{f^{\diamond}}{\sigma^{2}}+x y \frac{n}{\sigma} \ddot{\sigma} & =-x y(\lambda-c) f^{2} \\
x y\left(\frac{f^{\diamond}}{\sigma^{2}}+\frac{n}{\sigma} \ddot{\sigma}+(\lambda-c) f^{2}\right) & =0 .
\end{aligned}
$$


Then,

$$
\lambda=c-\frac{1}{f^{2}}\left(\frac{n}{\sigma} \ddot{\sigma}+\frac{f^{\diamond}}{\sigma^{2}}\right)
$$

and the proof is complete.

Theorem 25. Let $(\bar{M}, \bar{g}, \bar{\zeta}, \lambda)$ be a Ricci soliton where $\bar{M}={ }_{f} I \times_{\sigma} M$ is doubly warped spacetime and $\bar{\zeta}=h \partial_{t}+\zeta \epsilon$ $\mathfrak{X}(\bar{M})$ is a concurrent vector field on $\bar{M}$. Then,

(1) $(M, g)$ is Einstein manifold with factor $\mu=(\lambda-2) \sigma^{2}+$ $\sigma^{\diamond} / f^{2}$ if $f$ is constant,

(2) $\lambda=2-\left(1 / f^{2}\right)\left((n / \sigma) \ddot{\sigma}+f^{\diamond} / \sigma^{2}\right)$.

Let $\bar{\zeta}=h \partial_{t}+\zeta \in \mathfrak{X}(\bar{M})$, and then

$$
\begin{aligned}
\left(\overline{\mathscr{L}}_{\bar{\zeta}} \bar{g}\right)(\bar{X}, \bar{Y}) & \\
= & -2 x y f^{2}[\dot{h}+\zeta(\ln f)]+\sigma^{2}\left(\mathscr{L}_{\zeta} g\right)(X, Y) \\
& +2 h \sigma \dot{\sigma} g(X, Y),
\end{aligned}
$$

$$
\overline{\operatorname{Ric}}(\bar{X}, \bar{Y})
$$

$$
\begin{aligned}
= & x y\left(\frac{n \ddot{\sigma}}{\sigma}+\frac{f^{\diamond}}{\sigma^{2}}\right) \\
& +(n-1)\left(\frac{x \dot{\sigma}}{\sigma} Y(\ln f)+\frac{y \dot{\sigma}}{\sigma} X(\ln f)\right) \\
& +\operatorname{Ric}(X, Y)-\frac{1}{f} H^{f}(X, Y)-\frac{\sigma^{\diamond}}{f^{2}} g(X, Y) .
\end{aligned}
$$

Thus,

$$
\begin{aligned}
\frac{1}{2}\left(\overline{\mathscr{L}}_{\bar{\zeta}} \bar{g}\right)(\bar{X}, \bar{Y})+\overline{\operatorname{Ric}}(\bar{X}, \bar{Y}) \\
=-x y f^{2}[\dot{h}+\zeta(\ln f)]+\frac{1}{2} \sigma^{2}\left(\mathscr{L}_{\zeta} g\right)(X, Y) \\
\quad+h \sigma \dot{\sigma} g(X, Y)+x y\left(\frac{n \ddot{\sigma}}{\sigma}+\frac{f^{\diamond}}{\sigma^{2}}\right) \\
\quad+(n-1)\left(\frac{x \dot{\sigma}}{\sigma} Y(\ln f)+\frac{y \dot{\sigma}}{\sigma} X(\ln f)\right) \\
\quad+\operatorname{Ric}(X, Y)-\frac{1}{f} H^{f}(X, Y)-\frac{\sigma^{\diamond}}{f^{2}} g(X, Y)
\end{aligned}
$$

Suppose that $f=\sigma=1$ and $(M, g, \zeta, \dot{h})$ is a Ricci soliton on $M$, and then

$$
\begin{aligned}
\frac{1}{2} & \left(\overline{\mathscr{L}}_{\bar{\zeta}} \bar{g}\right)(\bar{X}, \bar{Y})+\overline{\operatorname{Ric}}(\bar{X}, \bar{Y}) \\
& =-x y \dot{h}+\frac{1}{2}\left(\mathscr{L}_{\zeta} g\right)(X, Y)+\operatorname{Ric}(X, Y) \\
& =-x y \dot{h}+\dot{h} g(X, Y)=\dot{h} \bar{g}(\bar{X}, \bar{Y}) .
\end{aligned}
$$

Therefore, $(\bar{M}, \bar{g}, \bar{\zeta}, \lambda)$ is a Ricci soliton where $\lambda=\dot{h}$. This discussion leads us to the following result.

Theorem 26. Let $\bar{M}={ }_{f} I \times{ }_{\sigma} M$ be doubly warped spacetime and $\bar{\zeta}=h \partial_{t}+\zeta \in \mathfrak{X}(\bar{M})$ be a vector field on $\bar{M}$. Then $(\bar{M}, \bar{g}, \bar{\zeta}, \lambda)$ is a Ricci soliton if

(1) $(M, g, \zeta, \dot{h})$ is a Ricci soliton on $M$,

(2) $f=\sigma=1$,

(3) $\lambda=\dot{h}$.

Let $f=1, \zeta$ be a conformal vector field with factor $2 \rho$, and $M$ be Einstein with factor $\mu$, and then

$$
\begin{aligned}
\frac{1}{2}(\overline{\mathscr{L}} \bar{\zeta} \bar{g})(\bar{X}, \bar{Y})+\overline{\operatorname{Ric}}(\bar{X}, \bar{Y}) \\
=-x y \dot{h}+\rho \sigma^{2} g(X, Y)+h \sigma \dot{\sigma} g(X, Y)+x y\left(\frac{n \ddot{\sigma}}{\sigma}\right) \\
\quad+\left(\mu-\sigma^{\diamond}\right) g(X, Y) \\
=-x y\left(\dot{h}-\frac{n \ddot{\sigma}}{\sigma}\right) \\
\quad+\left(\frac{\mu-\sigma^{\diamond}}{\sigma^{2}}+\rho+\frac{h}{\sigma} \dot{\sigma}\right) \sigma^{2} g(X, Y) ;
\end{aligned}
$$

that is, $(\bar{M}, \bar{g}, \bar{\zeta}, \lambda)$ is a Ricci soliton if

$$
\begin{aligned}
\dot{h}-\frac{n \ddot{\sigma}}{\sigma} & =\frac{\mu-\sigma^{\diamond}}{\sigma^{2}}+\rho+\frac{h}{\sigma} \dot{\sigma} \\
(\dot{h}-\rho) \sigma^{2} & =\mu+(n-1)\left(\sigma \ddot{\sigma}-\dot{\sigma}^{2}\right)+h \sigma \dot{\sigma} .
\end{aligned}
$$

Theorem 27. Let $\bar{M}=I_{f} \times_{\sigma} M$ be doubly warped spacetime and $\bar{\zeta}=h \partial_{t}+\zeta \in \mathfrak{X}(\bar{M})$ be a vector field on $\bar{M}$. Then, $(\bar{M}, \bar{g}, \bar{\zeta}, \lambda)$ is a Ricci soliton if

(1) $(M, g)$ is Einstein with factor $\mu$,

(2) $f=1$, and $\zeta$ is conformal with factor $2 \rho$,

(3) $(\dot{h}-\rho) \sigma^{2}=\mu+(n-1)\left(\sigma \ddot{\sigma}-\dot{\sigma}^{2}\right)+h \sigma \dot{\sigma}$.

In this case, $\lambda=\dot{h}-n \ddot{\sigma} / \sigma$.

\section{Appendix}

\section{Concurrent Vector Fields on Doubly Spacetime}

Let us now consider an example. Let $\bar{M}={ }_{f} I \times_{\sigma} \mathbb{R}$ be 2dimension doubly warped spacetime equipped with the metric $\bar{g}=-f^{2} d t^{2} \oplus \sigma^{2} d x^{2}$. Then,

$$
\begin{aligned}
& \bar{D}_{\partial_{t}} \partial_{t}=\frac{f f^{\prime}}{\sigma^{2}} \partial_{x} \\
& \bar{D}_{\partial_{x}} \partial_{t}=\frac{f^{\prime}}{f} \partial_{t}+\frac{\dot{\sigma}}{\sigma} \partial_{x}
\end{aligned}
$$




$$
\begin{aligned}
& \bar{D}_{\partial_{t}} \partial_{x}=\bar{D}_{\partial_{x}} \partial_{t} \\
& \bar{D}_{\partial_{x}} \partial_{x}=-\frac{\sigma \dot{\sigma}}{f^{2}} \partial_{t} .
\end{aligned}
$$

A vector field $\zeta=h \partial_{t}+k \partial_{x} \in \mathfrak{X}(\bar{M})$ is a concurrent vector field if

$$
\begin{aligned}
& \bar{D}_{\partial_{t}} \zeta=\partial_{t} \\
& \bar{D}_{\partial_{x}} \zeta=\partial_{x} .
\end{aligned}
$$

The first equation implies that

$$
\begin{aligned}
\bar{D}_{\partial_{t}}\left(h \partial_{t}+k \partial_{x}\right) & =\partial_{t} \\
\dot{h} \partial_{t}+\frac{h f f^{\prime}}{\sigma^{2}} \partial_{x}+k\left(\frac{f^{\prime}}{f} \partial_{t}+\frac{\dot{\sigma}}{\sigma} \partial_{x}\right) & =\partial_{t}
\end{aligned}
$$

and so

$$
\begin{aligned}
\dot{h} f+k f^{\prime} & =f \\
h f f^{\prime}+k \sigma \dot{\sigma} & =0 .
\end{aligned}
$$

Also, (A.3) implies that

$$
\begin{aligned}
\bar{D}_{\partial_{x}}\left(h \partial_{t}+k \partial_{x}\right) & =\partial_{x} \\
h\left(\frac{f^{\prime}}{f} \partial_{t}+\frac{\dot{\sigma}}{\sigma} \partial_{x}\right)+k^{\prime} \partial_{x}+k\left(-\frac{\sigma \dot{\sigma}}{f^{2}} \partial_{t}\right) & =\partial_{x}
\end{aligned}
$$

and so

$$
\begin{aligned}
h f f^{\prime}-k \sigma \dot{\sigma} & =0 \\
h \dot{\sigma}+k^{\prime} \sigma & =\sigma .
\end{aligned}
$$

By solving (A.6) and (A.8), we get $h f f^{\prime}=0$. Thus, $h=0$ or $f^{\prime}=0$. In both cases, $k \sigma \dot{\sigma}=0$; that is, $k=0$ or $\dot{\sigma}=0$. This discussion shows that we have the following cases using (A.5) and (A.9).

Case 1. $h=0$ and $\dot{\sigma}=0$ : then $k f^{\prime}=f$ and $k^{\prime} \sigma=\sigma$ and so $k=x+a \neq 0$ and

$$
\frac{f^{\prime}}{f}=\frac{1}{x+a}
$$

Therefore, $f=r(x+a)$, where both $r$ and $(x+a)$ are positive.

Case 2. $f^{\prime}=0$ and $k=0$ : then $\dot{h} f=f$ and $h \dot{\sigma}=\sigma$ and so $h=t+a \neq 0$ and similarly $\sigma=r(t+a)$ where both $r$ and $(t+a)$ are positive.

Case 3. $f^{\prime}=0$ and $\dot{\sigma}=0$ : then $\dot{h} f=f$ and $k^{\prime} \sigma=\sigma$ and so $h=t+a$ and $k=x+b$.

\section{Competing Interests}

The authors declare that there is no conflict of interests regarding the publication of this paper.

\section{References}

[1] R. L. Bishop and B. O’Neill, "Manifolds of negative curvature," Transactions of the American Mathematical Society, vol. 145, pp. $1-49,1969$.

[2] J. K. Beem and T. G. Powell, "Geodesic completeness and maximality in Lorentzian warped products," Tensor, vol. 39, pp. 31-36, 1982.

[3] D. E. Allison, "Geodesic completeness in static space-times," Geometriae Dedicata, vol. 26, no. 1, pp. 85-97, 1988.

[4] D. Allison, "Pseudoconvexity in Lorentzian doubly warped products," Geometriae Dedicata, vol. 39, no. 2, pp. 223-227, 1991.

[5] A. Gebarowski, "Doubly warped products with harmonic Weyl conformal curvature tensor," Colloquium Mathematicum, vol. 67, pp. 73-89, 1993.

[6] A. Gebarowski, "On conformally flat doubly warped products," Soochow Journal of Mathematics, vol. 21, no. 1, pp. 125-129, 1995.

[7] A. Gebarowski, "On conformally recurrent doubly warped products," Tensor, vol. 57, no. 2, pp. 192-196, 1996.

[8] B. Ünal, "Doubly warped products," Differential Geometry and Its Applications, vol. 15, no. 3, pp. 253-263, 2001.

[9] M. Faghfouri and A. Majidi, "On doubly warped product immersions," Journal of Geometry, vol. 106, no. 2, pp. 243-254, 2015.

[10] A. Olteanu, "A general inequality for doubly warped product submanifolds," Mathematical Journal of Okayama University, vol. 52, pp. 133-142, 2010.

[11] A. Olteanu, "Doubly warped products in S-space forms," Romanian Journal of Mathematics and Computer Science, vol. 4, no. 1, pp. 111-124, 2014.

[12] S. Y. Perktas and E. Kilic, "Biharmonic maps between doubly warped product manifolds," Balkan Journal of Geometry and Its Applications, vol. 15, no. 2, pp. 159-170, 2010.

[13] V. N. Berestovskii and Yu. G. Nikonorov, "Killing vector fields of constant length on Riemannian manifolds," Siberian Mathematical Journal, vol. 49, no. 3, pp. 395-407, 2008.

[14] S. Deshmukh and F. R. Al-Solamy, "Conformal vector fields on a Riemannian manifold," Balkan Journal of Geometry and Its Applications, vol. 19, no. 2, pp. 86-93, 2014.

[15] S. Deshmukh and F. Al-Solamy, "A note on conformal vector fields on a Riemannian manifold," Colloquium Mathematicum, vol. 136, no. 1, pp. 65-73, 2014.

[16] W. Kuhnel and H.-B. Rademacher, "Conformal vector fields on pseudo-Riemannian spaces," Differential Geometry and Its Applications, vol. 7, no. 3, pp. 237-250, 1997.

[17] M. Sánchez, "On the geometry of generalized RobertsonWalker spacetimes: curvature and Killing fields," Journal of Geometry and Physics, vol. 31, no. 1, pp. 1-15, 1999.

[18] M. Steller, "Conformal vector fields on spacetimes," Annals of Global Analysis and Geometry, vol. 29, no. 4, pp. 293-317, 2006.

[19] B. O'Neill, Semi-Riemannian Geometry with Applications to Relativity, Academic Press Limited, London, UK, 1983.

[20] Y. Agaoka, I.-B. Kim, B. H. Kim, and D. J. Yeom, "On doubly warped product manifolds," Memoirs of the Faculty of Integrated Arts and Sciences Hiroshima University, Series IV, vol. 24, pp. 110, 1998.

[21] M. P. Ramos, E. G. Vaz, and J. Carot, "Double warped spacetimes," Journal of Mathematical Physics, vol. 44, no. 10, pp. 48394865, 2003.

[22] B. Unal, Doubly warped products [Ph.D. thesis], University of Missouri, Columbia, Mo, USA, 2000. 
[23] B.-Y. Chen and S. Deshmukh, "Ricci solitons and concurrent vector fields," Balkan Journal of Geometry and Its Applications, vol. 20, no. 1, pp. 14-25, 2015.

[24] F. Dobarro and B. Ünal, "Characterizing Killing vector fields of standard static space-times," Journal of Geometry and Physics, vol. 62, no. 5, pp. 1070-1087, 2012.

[25] S. Shenawy and B. Ünal, "2-Killing vector fields on warped product manifolds," International Journal of Mathematics, vol. 26, no. 9, Article ID 1550065, 17 pages, 2015.

[26] G. S. Hall, Symmetries and Curvature Structure in General Relativity, World Scientific, Singapore, 2004.

[27] J. Carot, J. da Costa, and E. G. Vaz, "Matter collineations: the inverse 'symmetry inheritance' problem," Journal of Mathematical Physics, vol. 35, no. 9, pp. 4832-4838, 1994.

[28] P. S. Apostolopoulos and J. G. Carot, "Conformal symmetries in warped manifolds," Journal of Physics: Conference Series, vol. 8, pp. 28-33, 2005.

[29] J. Carot and B. O. J. Tupper, "Conformally reducible $2+2$ spacetimes," Classical and Quantum Gravity, vol. 19, no. 15, pp. 4141-4166, 2002.

[30] J. Carot, A. J. Keane, and B. O. J. Tupper, "Conformally reducible 1+3 spacetimes," Classical and Quantum Gravity, vol. 25, no. 5, Article ID 055002, 2008.

[31] N. Van den Bergh, "Conformally $2+2$-decomposable perfect fluid spacetimes with constant curvature factor manifolds," Classical and Quantum Gravity, vol. 29, no. 23, Article ID 235003, 2012.

[32] A. Barros and E. Ribeiro Jr., "Some characterizations for compact almost Ricci solitons," Proceedings of the American Mathematical Society, vol. 140, no. 3, pp. 1033-1040, 2012.

[33] A. Barros, J. N. Gomes, and E. Ribeiro Jr., "A note on rigidity of the almost Ricci soliton," Archiv der Mathematik, vol. 100, no. 5, pp. 481-490, 2013.

[34] M. Fernández-López and E. García-Río, "Rigidity of shrinking Ricci solitons," Mathematische Zeitschrift, vol. 269, no. 1-2, pp. 461-466, 2011.

[35] O. Munteanu and N. Sesum, "On gradient Ricci solitons," Journal of Geometric Analysis, vol. 23, no. 2, pp. 539-561, 2013.

[36] P. Petersen and W. Wylie, "Rigidity of gradient Ricci solitons," Pacific Journal of Mathematics, vol. 241, no. 2, pp. 329-345, 2009. 


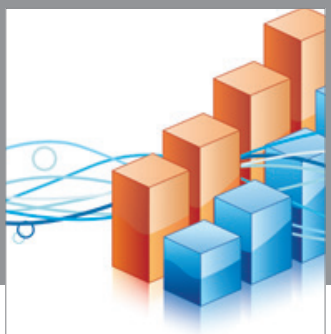

Advances in

Operations Research

vatem alat4

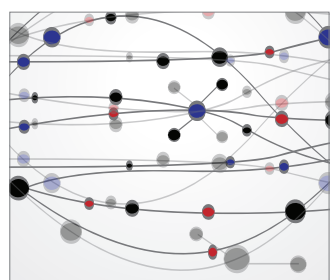

\section{The Scientific} World Journal
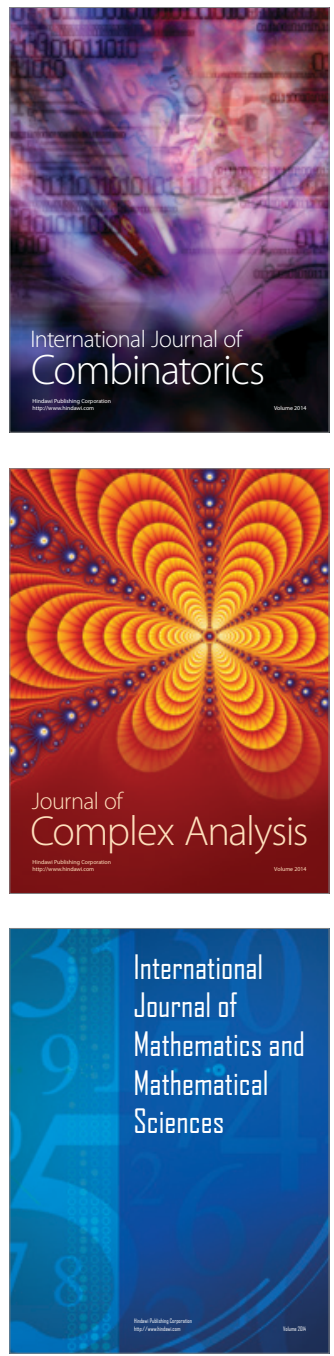
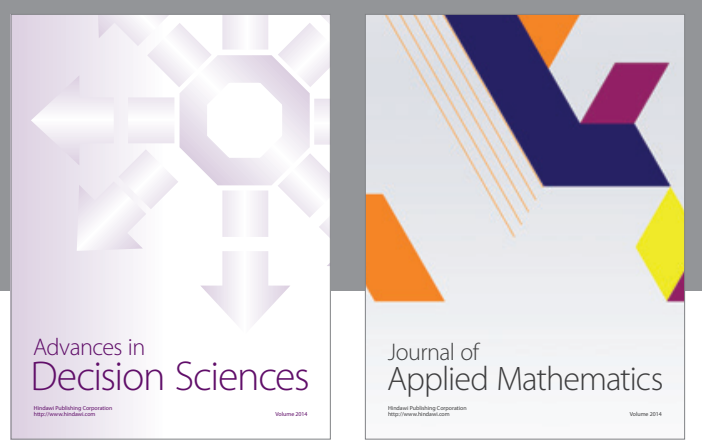

Algebra

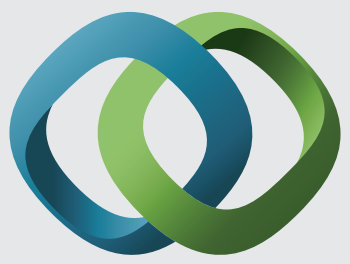

\section{Hindawi}

Submit your manuscripts at

http://www.hindawi.com
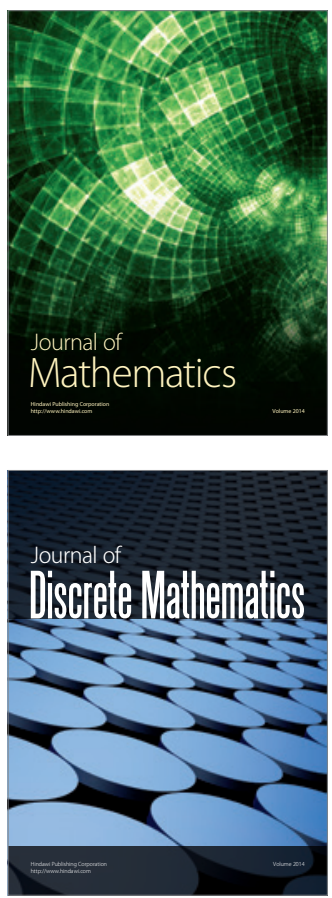

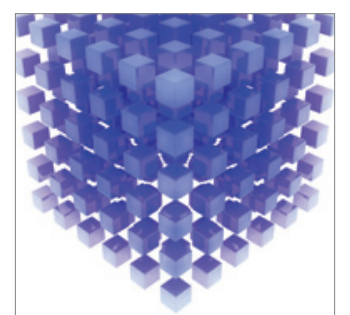

Mathematical Problems in Engineering
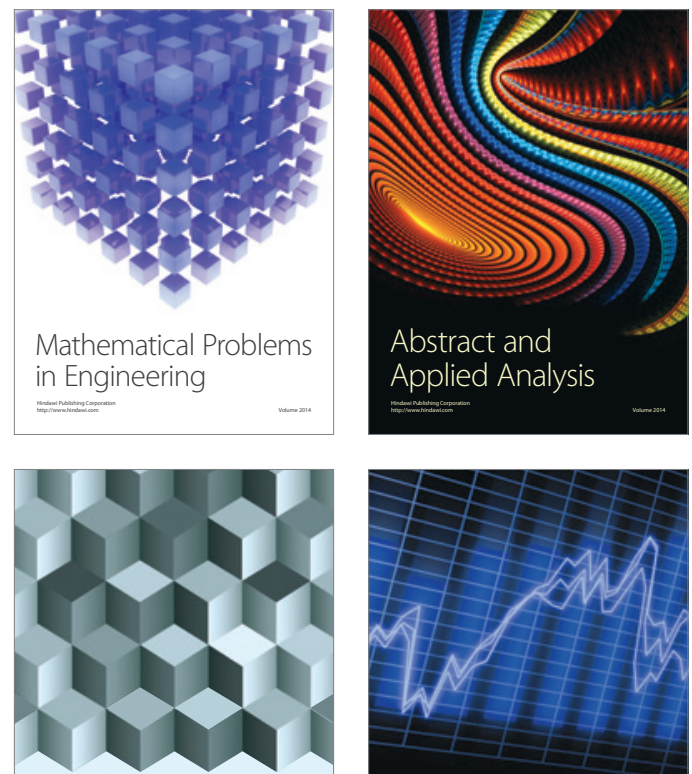

Journal of

Function Spaces

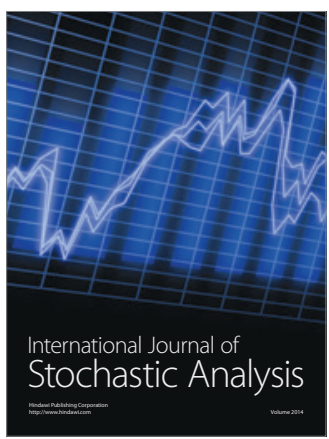

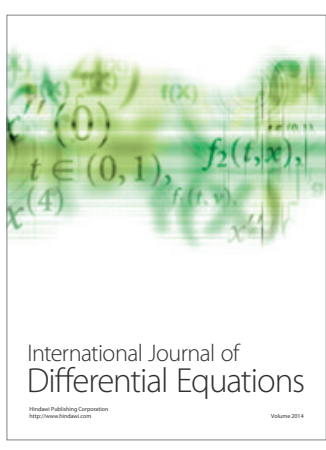
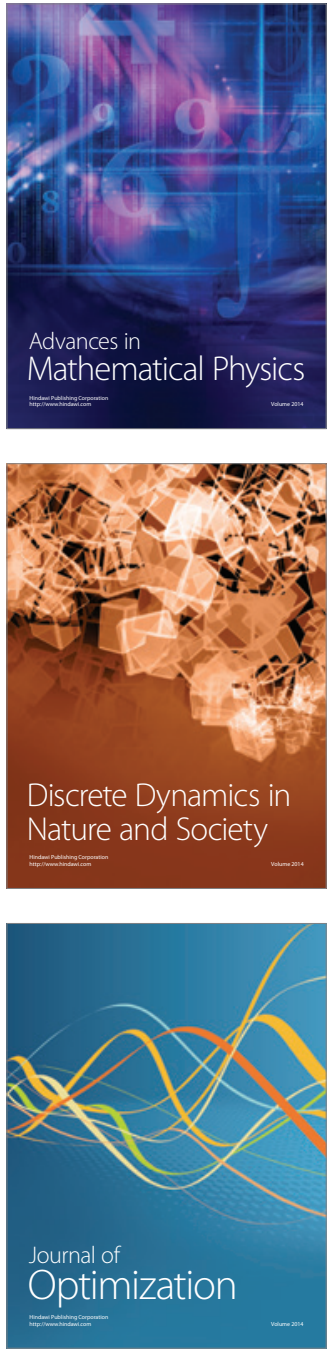